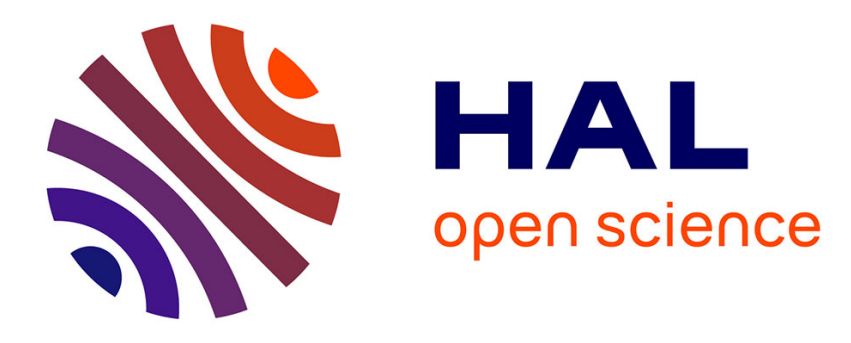

\title{
Dynamic modelling of a two-wheeled vehicle: Jourdain formalism
}

\author{
Lamri Nehaoua, Hichem Arioui, Nicolas Seguy, Saïd Mammar
}

\section{To cite this version:}

Lamri Nehaoua, Hichem Arioui, Nicolas Seguy, Saïd Mammar. Dynamic modelling of a twowheeled vehicle: Jourdain formalism. Vehicle System Dynamics, 2013, 51 (5), pp.648- 670. 10.1080/00423114.2012.762536 . hal-00786265

\section{HAL Id: hal-00786265 \\ https://hal.science/hal-00786265}

Submitted on 8 Feb 2013

HAL is a multi-disciplinary open access archive for the deposit and dissemination of scientific research documents, whether they are published or not. The documents may come from teaching and research institutions in France or abroad, or from public or private research centers.
L'archive ouverte pluridisciplinaire HAL, est destinée au dépôt et à la diffusion de documents scientifiques de niveau recherche, publiés ou non, émanant des établissements d'enseignement et de recherche français ou étrangers, des laboratoires publics ou privés. 


\title{
Dynamic Modeling of a Two Wheeled Vehicle : Jourdain Formalism
}

\author{
L. Nehaoua*, H. Arioui, N. Seguy and S. Mammar* \\ IBISC Laboratory, 40 rue de pelvoux, 91020 Evry Cedex, France
}

February 8, 2013

\begin{abstract}
This paper presents a motorcycle direct dynamic formulation by the Jourdain's principle approach. This vehicle is considered as an assembly of six rigid bodies and the resulting equation of motion allows to simulate 11 Degrees of Freedom $(\mathrm{DoF})$. The vehicle geometry is described and a step by step procedure is introduced to evaluate the kinematics and the generalized efforts of the considered vehicle. In addition, to simulate the equation of motion, a Lyapunov based stabilization is developed to assess the vehicle behavior in response to a propulsion/braking torque applied on the vehicle's wheels and a rider torque exerted on the motorcycle's handlebar. Simulation results reveal some dynamics features like load transfer and counter-steering phenomena.
\end{abstract}

\section{keywords}

Motorcycle modeling, motorcycle control, Jourdain's dynamics principle.

\section{Introduction}

During last decades, motorcycles have become a popular transportation tool. Indeed, the number of two-wheeled vehicles is in constant increase, specifically in urban areas. However, the risk associated with driving such vehicles is important and a road accident, even trivial, can have fatal consequences on the two-wheeled users.

Motorcyclists are the most vulnerable road users. They represent less than $2 \%$ of the traffic, but $18 \%$ of those killed in 2010 in France [1]. There are many causes to these fatalities: alcohol, non-compliance with the road rules, poor visibility, lack of assistive devices and especially the overvaluation of the self mastering capabilities of such a vehicle. Indeed, with the same power, the motorcycle represents more aggressive dynamics compared to those of a car vehicle. Riding a two-wheeled vehicle is neither simple nor intuitive like driving a car vehicle. Involved accelerations, critical equilibrium, trajectory anticipation are, among others, many points that make riding a

**Corresponding author. Email: lamri.nehaoua@iup.univ-evry.fr 
motorcycle a complicated exercise especially for inexperienced riders who get carried away by the riding sensations and pleasure.

Fortunately, recent preventive as well as suppressive laws have significantly contributed to decrease number of fatalities. It must be said that motorcyclists' road safety has attracted the attention of all academic, industrial and political institutions. In France, several research projects were undertaken: SUMOTORI ${ }^{1}$ (2003-2006), SIMA$\mathrm{COM}^{2}$ (2006-2009) [2], SAFERIDER ${ }^{3}$ (2008-2010), DAMOTO ${ }^{4}$ (2008-2011), and others with a common point: understand the rider behavior.

To deal with this aim, it is necessary to predict the dynamics of the motorcycle vehicle and model it. In the study of mechanical systems, modeling usually leads to a set of differential/algebraic equations (DAE) derived from laws of mechanics. In this field, two approaches are proposed: the analytical approach and the numerical one. In the later one, many tools were developed such MSC ADAMS [3], simMechanics [4] and Modelica [5]. The alternative approach is the development of symbolic equations of motion which is a time consuming method, but it offers more flexibilities in the system description. In that case, an algebra package like MBsymba [6] is highly recommended.

Many works have addressed the modeling of the two-wheeled vehicles. Indeed, the first study on the stability and balance of a bicycle vehicle was performed by Whipple [7]. This bicycle is represented by two bodies linked via the steering mechanism. Wheels are modeled as circular thin disks where the gyroscopic effect is considered. Other research works have been undertaken $[8,9,10]$. In all these studies, the tireroad interaction has been neglected. In 1971, Sharp integrated tires' efforts [11]. The objective of this study is to examine the effect of parameters uncertainties on the vehicle stability. The vehicle is modeled as a set of two rigid bodies connected by the steering mechanism, simulating 4 DoF: the lateral displacement and the yaw, the roll and the steering rotations.

So far, in all these models, the longitudinal velocity was assumed to be constant and the vehicle suspension motions were ignored. It was interesting to examine how these factors influenced the frequency characteristics of the different stability modes. These aspects have been investigated in a further work of Sharp in 1974 [12]. In 1994, Sharp published a paper, in which, the motorcycle is represented by a set of four rigid bodies to simulate $8 \mathrm{DoF}$. The originality of this model lies in the integration of flexibilities and the rider tilting motion [13].

In the same perspective, a model incorporating the suspension travels and a more complex representation of the tire-road interaction is presented in [14]. The advances made by this model have allowed exploring the dynamics of such a vehicle for a large motion around equilibrium. In that case, the interaction between the in-plane and the out-of-plane motion is well described. More recently, the FastBike program presents a nonlinear model of a motorcycle described as a set of 6 bodies and simulating 11 DoF. Each body is identified by its natural coordinates (a vector of six coordinates: three Cartesian coordinates of its center of mass and three rotations) and the assembly

\footnotetext{
${ }^{1}$ Safety of motorcyclist against risky environment

${ }^{2}$ Simulator for learning to drive motorized two-wheeled vehicles

${ }^{3}$ Advanced telematics for enhancing the SAFETY and comfort of motorcycle RIDERS

${ }^{4}$ Detection of accident situations on motorcycle
} 
is done by introducing geometric constraints [15]. Finally, a modeling technique based on the recursive Newton-Euler approach is adapted to derive a motorcycle dynamics model intended for a two-wheeled simulator application $[16,17]$.

Among the vehicle models, the simulation of single-track one turns out to be particularly difficult. While a car vehicle remains stable, the stabilization of two-wheeled vehicles is required and a rider model must be included. The literature of two-wheeled vehicle stabilization is recent $[19,18,20]$. Generally, a two-layer controller is adopted to track the reference road trajectory and an inner controller stabilizes the longitudinal and lateral dynamics. For this, several strategies are used: the optimal maneuver method [21, 22], robust $\mathcal{H}_{\infty}$ and LPV ${ }^{1}$ control techniques [23].

In this paper, a step by step modeling approach based on the Jourdain's principle is presented. This method was firstly used in [24] to derive a car dynamics model and the modeling prerequisites are inspired from this work. It offers an efficient tool for deriving motion equations in a systematic way and with less theoretical skills comparing to the Lagrangian and the Newton/Euler approaches. The great efforts concern the computation of Jacobian matrices and the virtual power of the different external applied forces. Our aim is to derive a mathematical description of a motorcycle vehicle with middle complexity, which captivates the essential dynamics issues. This model is intended for control, state observation and security devices development applications. In section 2, a brief outline to the mathematical modeling of the motorcycle kinematics and dynamics is introduced. Section 3 focuses on the computation of the generalized vector efforts. Section 4 is intended to demonstrate the basics concepts of the tire/road contact. Finally, a virtual rider controller is introduced in order to regulate the longitudinal speed towards a reference profile and to stabilize the lateral dynamics by using the roll motion.

\section{Motorcycle kinematics and dynamics}

\subsection{Motorcycle description}

In the present work, the motorcycle is composed of six bodies (see Figure 1). A rear body $G_{r}$ including the saddle, the engine and the fuel tank. The upper front body $G_{f}$ includes the handlebar and the upper part of the suspension assembly. The lower front body $G_{l}$ represents the lower part of the suspension assembly and the brake system. The swing arm body $G_{s}$ contains the swing arm mass and the rear brake system. Finally, $R_{f}$ and $R_{r}$ represent respectively, the front and the rear wheel bodies.

Most of these bodies are connected via revolute link except the upper and lower parts of the front body which are interconnected with a prismatic link (telescopic suspension). In order to completely define the configuration of the motorcycle, a set of 11 DoF has been considered. A full characterization of motorcycle's kinematics includes :

- the longitudinal, lateral and vertical position $(x, y, z)$ of the rear body,

- the roll, pitch and yaw orientation $(\varphi, \theta, \psi)$ of the rear body,

${ }^{1}$ LPV: Linear Parameter Varying 
- the steering angle $\delta$ of the front body and the front wheel,

- the front suspension travel $\lambda_{f}$ and the swing arm rotation $\mu$,

- and finally, the front and rear wheels spinning, $\zeta_{f}$ and $\zeta_{r}$.

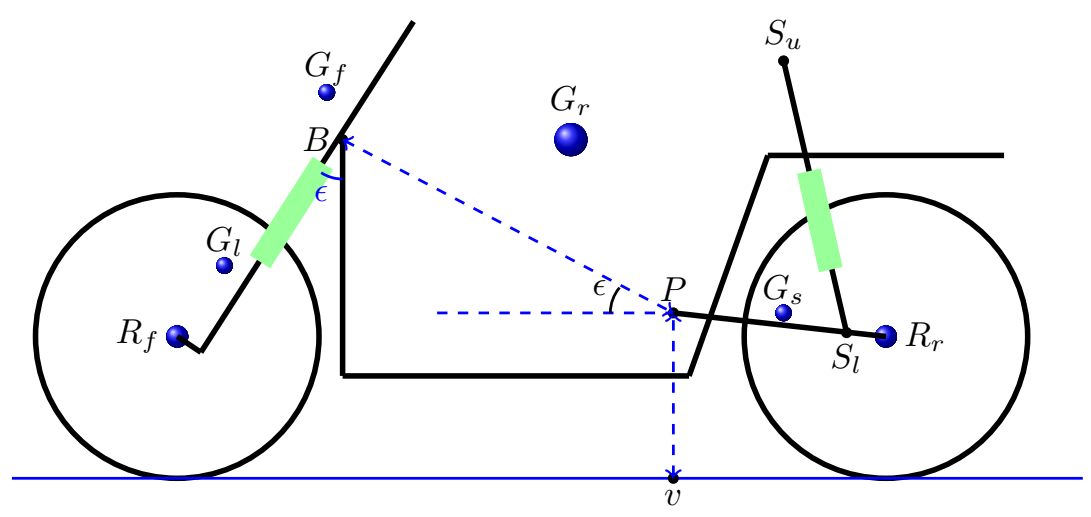

Figure 1: The motorcycle geometrical description

\subsection{Kinematic background}

Consider a right-handed, orthogonal axis reference frame $\Re_{v}\left(v, \boldsymbol{i}_{v}, \boldsymbol{j}_{v}, \boldsymbol{k}_{v}\right)$ attached to the vehicle at point $v$ in which the motion of the overall mechanical system will be expressed. Point $v$ is defined as the intersection of the longitudinal plane of symmetry, the ground plane, and the vertical transverse plane containing point $P$, attach point of the swing arm with the rear body. The motion of the motorcycle is referred to the inertial reference frame $\Re_{o}\left(O, \boldsymbol{i}_{o}, \boldsymbol{j}_{o}, \boldsymbol{k}_{o}\right)$, by the velocity vector components $v_{x}, v_{y}$ of point $v$, and the yaw rotation $\psi$ around the z-axis $\boldsymbol{k}_{o}$ of the inertial reference frame $\Re_{o}$ (see Figure 2). Starting from the reference frame $\Re_{v}$, the orientations of the other reference frames are defined as follows (see Figure 3). A roll rotation $\varphi$ about $\boldsymbol{i}_{v}$ gives $\Re_{\varphi}\left(v, \boldsymbol{i}_{\varphi}, \boldsymbol{j}_{\varphi}, \boldsymbol{k}_{\varphi}\right)$. Next, a pitch rotation $\theta$ followed by a castor rotation $\epsilon$ about $\boldsymbol{j}_{\varphi}$ gives $\Re_{\epsilon}\left(P, \boldsymbol{i}_{\epsilon}, \boldsymbol{j}_{\epsilon}, \boldsymbol{k}_{\epsilon}\right)$. Then, a steer rotation $\delta$ about $\boldsymbol{k}_{\epsilon}$ gives $\Re_{\delta}\left(B, \boldsymbol{i}_{\delta}, \boldsymbol{j}_{\delta}, \boldsymbol{k}_{\delta}\right)$. In the other side, a swing rotation $\mu$ about $\boldsymbol{j}_{\varphi}$ gives $\Re_{\mu}\left(P, \boldsymbol{i}_{\mu}, \boldsymbol{j}_{\mu}, \boldsymbol{k}_{\mu}\right)$. 


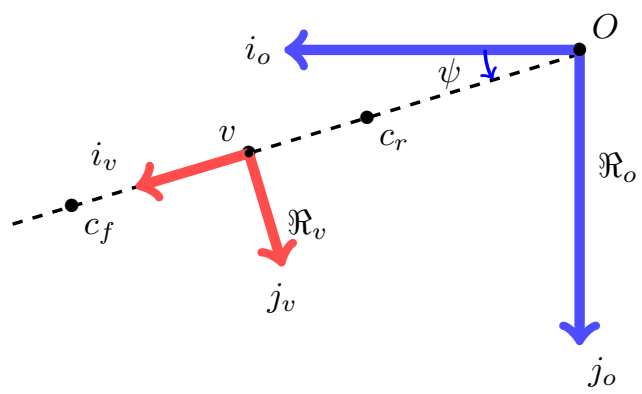

Figure 2: Configuration of the vehicle system axis $\Re_{v}$ w.r.t the inertial reference frame $\Re_{o}$

In the movable reference frame $\Re_{v}$, the position of a body $i$ is given by:

$$
\boldsymbol{r}_{o i}=\boldsymbol{r}_{o v}+\boldsymbol{r}_{v i}
$$

From equation (1), the velocity and the acceleration of a body $i$ are derived as following:

$$
\begin{aligned}
& \boldsymbol{v}_{o i}=\boldsymbol{v}_{o v}+\boldsymbol{\omega}_{o v} \times \boldsymbol{r}_{v i}+\dot{\boldsymbol{r}}_{v i} \\
& \boldsymbol{a}_{o i}=\boldsymbol{a}_{o v}+\boldsymbol{\epsilon}_{o v} \times \boldsymbol{r}_{v i}+\boldsymbol{\omega}_{o v} \times\left(\boldsymbol{\omega}_{o v} \times \boldsymbol{r}_{v i}+2 \dot{\boldsymbol{r}}_{v i}\right)+\ddot{\boldsymbol{r}}_{v i}
\end{aligned}
$$

and, the angular velocity $\boldsymbol{\omega}_{o i}$ and the angular acceleration $\boldsymbol{\epsilon}_{o i}$ vectors of body $i$ are derived in the same way:

$$
\begin{aligned}
\boldsymbol{\omega}_{o i} & =\boldsymbol{\omega}_{o v}+\boldsymbol{\omega}_{v i} \\
\boldsymbol{\epsilon}_{o i} & =\boldsymbol{\epsilon}_{o v}+\boldsymbol{\omega}_{o v} \times \boldsymbol{\omega}_{v i}+\dot{\boldsymbol{\omega}}_{v i}
\end{aligned}
$$

Since the kinematic configuration of each body is well defined in its local reference frame, it remains to define the kinematic configuration of the movable reference frame $\Re_{v}$, it means, to find the expressions of $\boldsymbol{a}_{o v}$ and $\boldsymbol{\epsilon}_{o v}$ in equations $(2,3)$ w.r.t $\dot{\boldsymbol{v}}_{o v}$ and $\dot{\boldsymbol{\omega}}_{o v}$. Suppose that the linear and angular velocity vectors of $\Re_{v}$ w.r.t the inertial one $\Re_{o}$ are given by $\boldsymbol{v}_{o v}^{o}=\mathbf{R}_{v o} \boldsymbol{v}_{o v}^{v}$ and $\boldsymbol{\omega}_{o v}^{o}=\mathbf{R}_{v o} \boldsymbol{\omega}_{o v}^{v}$. It goes that the linear and angular acceleration vectors of $\Re_{v}$, expressed in $\Re_{v}$, can be derived as following:

$$
\begin{aligned}
\boldsymbol{a}_{o v} & =\boldsymbol{\omega}_{o v} \times \boldsymbol{v}_{o v}+\dot{\boldsymbol{v}}_{o v} \\
\boldsymbol{\epsilon}_{o v} & =\dot{\boldsymbol{\omega}}_{o v}
\end{aligned}
$$




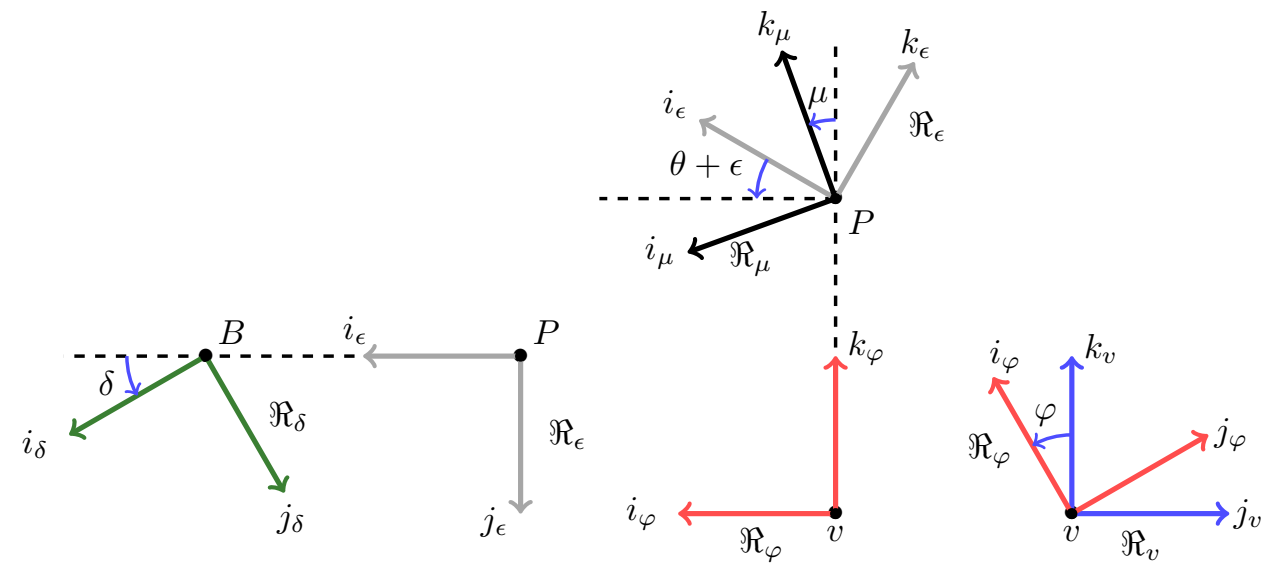

Figure 3: Axis systems and angular displacements configuration

From equations (2-4), the linear and angular acceleration vectors of each body $i$ are expressed in the movable reference frame $\Re_{v}$ by the following equations:

$$
\begin{aligned}
\boldsymbol{a}_{o i} & =\dot{\boldsymbol{v}}_{o v}+\dot{\boldsymbol{\omega}}_{o v} \times \boldsymbol{r}_{v i}+\ddot{\boldsymbol{r}}_{v i}+\boldsymbol{\omega}_{o v} \times\left(\boldsymbol{v}_{o i}+\dot{\boldsymbol{r}}_{v i}\right) \\
\boldsymbol{\epsilon}_{o i} & =\dot{\boldsymbol{\omega}}_{o v}+\boldsymbol{\omega}_{o v} \times \boldsymbol{\omega}_{v i}+\dot{\boldsymbol{\omega}}_{v i}
\end{aligned}
$$

At present, all kinematic quantities are defined, then we introduce the principle of the virtual power called also Jourdain's principle.

\subsection{Jourdain's principle}

Jourdain's principle states that the virtual power done by motion compatible constraint efforts $\boldsymbol{F}_{i, c} / \boldsymbol{M}_{i, c}$ is null [24], then:

$$
\sum_{i=1}^{n}\left\{\left(\Delta \boldsymbol{v}_{o i}\right) \cdot \boldsymbol{F}_{i, c}+\left(\Delta \boldsymbol{\omega}_{o i}\right) \cdot \boldsymbol{M}_{i, c}\right\}=0
$$

where $n$ is the number of bodies and $\Delta \boldsymbol{v}$ describes a virtual velocity. By introducing the Newton/Euler dynamics principle, the constraint efforts $\boldsymbol{F}_{i, c} / \boldsymbol{M}_{i, c}$ can be expressed from the system motion and the external applied efforts $\boldsymbol{F}_{i, a} / \boldsymbol{M}_{i, a}$ as:

$$
\begin{aligned}
& \boldsymbol{F}_{i, c}=m_{i} \boldsymbol{a}_{o i}-\boldsymbol{F}_{i, a} \\
& \boldsymbol{M}_{i, c}=\mathbf{I}_{i} \boldsymbol{\epsilon}_{o i}+\boldsymbol{\omega}_{o i} \times \mathbf{I}_{i} \boldsymbol{\omega}_{o i}-\boldsymbol{M}_{i, a}
\end{aligned}
$$

Moreover, from equations $(2,3,5)$, the velocity and acceleration vectors can be written as following: 
$\boldsymbol{v}_{o i}=\frac{\partial \boldsymbol{v}_{o i}}{\partial \boldsymbol{\vartheta}} \boldsymbol{\vartheta} \quad \boldsymbol{\omega}_{o i}=\frac{\partial \boldsymbol{\omega}_{o i}}{\partial \boldsymbol{\vartheta}} \boldsymbol{\boldsymbol { a }} \quad \boldsymbol{a}_{o i}=\frac{\partial \boldsymbol{v}_{o i}}{\partial \boldsymbol{\vartheta}} \dot{\boldsymbol{\vartheta}}+\boldsymbol{a}_{R} \quad \boldsymbol{\epsilon}_{o i}=\frac{\partial \boldsymbol{\omega}_{o i}}{\partial \boldsymbol{\vartheta}} \dot{\boldsymbol{\vartheta}}+\boldsymbol{\epsilon}_{R}$

where:

$$
\boldsymbol{\vartheta}=\left[\begin{array}{lllllllllll}
v_{x} & v_{y} & \dot{\psi} & \dot{z} & \dot{\varphi} & \dot{\theta} & \dot{\delta} & \dot{\lambda}_{f} & \dot{\mu} & \dot{\zeta}_{f} & \dot{\zeta}_{r}
\end{array}\right]^{T}
$$

denotes the vector of generalized velocities and the partial derivatives are called Jacobian matrices of the velocity vector w.r.t the generalized velocities vector $\boldsymbol{\vartheta}$. Vectors $\boldsymbol{a}_{R}$ and $\boldsymbol{\epsilon}_{R}$ are referred as the residual acceleration terms. Consequently, from equations $(7,8)$ and after some algebraic manipulations, the motion dynamics can be expressed as:

$$
\mathbf{M} \dot{\vartheta}=Q
$$

where $\mathbf{M}$ is the mass matrix obtained from the direct computation of Jacobian matrices:

$$
\mathbf{M}=\sum_{i=1}^{n}\left\{m_{i}\left(\frac{\partial \boldsymbol{v}_{o i}}{\partial \boldsymbol{\vartheta}}\right)^{T} \frac{\partial \boldsymbol{v}_{o i}}{\partial \boldsymbol{\vartheta}}+\left(\frac{\partial \boldsymbol{\omega}_{o i}}{\partial \boldsymbol{\vartheta}}\right)^{T} \mathbf{I}_{i} \frac{\partial \boldsymbol{\omega}_{o i}}{\partial \boldsymbol{\vartheta}}\right\}
$$

and the generalized efforts vector $Q$ can be split as following:

$$
\begin{aligned}
\boldsymbol{Q} & =\underbrace{\sum_{i=1}^{n}\left\{\left(\frac{\partial \boldsymbol{v}_{o i}}{\partial \boldsymbol{\vartheta}}\right)^{T} \boldsymbol{F}_{i, a}+\left(\frac{\partial \boldsymbol{\omega}_{o i}}{\partial \boldsymbol{\vartheta}}\right)^{T} \boldsymbol{M}_{i, a}\right\}}_{\boldsymbol{Q}_{a}}- \\
& \underbrace{\sum_{i=1}^{n}\left\{m_{i}\left(\frac{\partial \boldsymbol{v}_{o i}}{\partial \boldsymbol{\vartheta}}\right) \boldsymbol{a}_{R}+\left(\frac{\partial \boldsymbol{\omega}_{o i}}{\partial \boldsymbol{\vartheta}}\right)^{T}\left(\mathbf{I}_{i} \boldsymbol{\epsilon}_{R}+\boldsymbol{\omega}_{o i} \times \mathbf{I}_{i} \boldsymbol{\omega}_{o i}\right)\right\}}_{\boldsymbol{Q}_{R}}
\end{aligned}
$$

The next subsection presents a step-by-step procedure to derive the Jacobian matrices. These matrices allow the calculation of the mass matrix $\mathbf{M}$ and the residual generalized effort vector $Q$.

\subsection{Motorcycle Kinematics}

\subsubsection{Rear body}

The rear body $G_{r}$ has 3 DoF w.r.t $\Re_{v}$ which are the roll, pitch rotations and vertical displacement. Then, its center of mass position vector is:

$$
\boldsymbol{r}_{v G_{r}}=\boldsymbol{r}_{v P}+\boldsymbol{r}_{P G_{r}}
$$


where the coordinates of vectors $\boldsymbol{r}_{v P}$ and $\boldsymbol{r}_{P G_{r}}$ in the reference frame $\Re_{v}$ are computed by:

$$
\boldsymbol{r}_{v P}=\mathbf{R}_{\varphi}\left[\begin{array}{c}
0 \\
0 \\
z+h_{p}
\end{array}\right] \quad \boldsymbol{r}_{P G_{r}}=\mathbf{R}_{\varphi, \theta}\left[\begin{array}{c}
x_{G r} \\
0 \\
z_{G r}
\end{array}\right]
$$

By differentiating, the relative linear and angular velocity vectors are:

$$
\begin{aligned}
\dot{\boldsymbol{r}}_{v G_{r}} & =\dot{\boldsymbol{r}}_{v P}+\dot{\boldsymbol{r}}_{P G_{r}}=\left(\boldsymbol{\omega}_{\varphi} \times \boldsymbol{r}_{v P}+\dot{z} \boldsymbol{k}_{\varphi}\right)+\boldsymbol{\omega}_{\varphi, \theta} \times \boldsymbol{r}_{P G_{r}} \\
\boldsymbol{\omega}_{v G_{r}} & =\boldsymbol{\omega}_{\varphi, \theta}=\boldsymbol{\omega}_{\varphi}+\boldsymbol{\omega}_{\theta}
\end{aligned}
$$

where the coordinates of the unit vector $\boldsymbol{k}_{\varphi}$ in $\Re_{v}$ are obtained by $\boldsymbol{k}_{\varphi}=\mathbf{R}_{\varphi}[0,0,1]^{T}$ and the components of the angular velocity vectors $\boldsymbol{\omega}_{\varphi}$ and $\boldsymbol{\omega}_{\theta}$ are respectively given by $\boldsymbol{\omega}_{\varphi}=[\dot{\varphi}, 0,0]^{T}, \boldsymbol{\omega}_{\theta}=\mathbf{R}_{\varphi}[0, \dot{\theta}, 0]^{T}$. By using equations $(5,8)$, the Jacobian matrices and the residual acceleration vectors can be deduced by simple algebraic manipulations.

\subsubsection{Front upper body}

The front upper body $G_{f}$ is belonging to the fixed-body reference frame $\Re_{\delta}$ and has 1 DoF w.r.t the rear body which represents the steer rotation. Then, its center of mass position vector is governed by:

$$
\boldsymbol{r}_{v G_{f}}=\boldsymbol{r}_{v P}+\boldsymbol{r}_{P B}+\boldsymbol{r}_{B G_{f}}
$$

where the coordinates of the vectors $\boldsymbol{r}_{P B}$ and $\boldsymbol{r}_{B G_{f}}$ are given in the reference frame $\Re_{v}$ as following:

$$
\boldsymbol{r}_{P B}=\mathbf{R}_{\varphi, \theta, \epsilon}\left[\begin{array}{c}
l_{P B} \\
0 \\
0
\end{array}\right] \quad \boldsymbol{r}_{B G_{f}}=\mathbf{R}_{\varphi, \theta, \epsilon, \delta}\left[\begin{array}{c}
x_{G f} \\
0 \\
z_{G f}
\end{array}\right]
$$

By differentiating, the relative linear and angular velocity vectors are:

$$
\begin{aligned}
\dot{\boldsymbol{r}}_{v G_{f}} & =\dot{\boldsymbol{r}}_{v P}+\dot{\boldsymbol{r}}_{P B}+\dot{\boldsymbol{r}}_{B G_{f}}=\dot{\boldsymbol{r}}_{v P}+\boldsymbol{\omega}_{\varphi, \theta} \times \boldsymbol{r}_{P B}+\boldsymbol{\omega}_{\varphi, \theta, \delta} \times \boldsymbol{r}_{B G_{f}} \\
\boldsymbol{\omega}_{v G_{f}} & =\boldsymbol{\omega}_{\varphi, \theta, \delta}=\boldsymbol{\omega}_{v G_{r}}+\boldsymbol{\omega}_{\delta}
\end{aligned}
$$

where $\boldsymbol{\omega}_{\delta}=\mathbf{R}_{\varphi, \theta, \epsilon}[0,0, \dot{\delta}]^{T}$. 


\subsubsection{Lower front body}

The front lower body has $1 \mathrm{DoF}$ w.r.t $G_{f}$ representing the suspension travel $\lambda_{f}$. Its center of mass position vector is:

$$
\boldsymbol{r}_{v G_{l}}=\boldsymbol{r}_{v B}+\boldsymbol{r}_{B G_{l}}
$$

where the coordinates of the vector $\boldsymbol{r}_{B G_{l}}$ are:

$$
\boldsymbol{r}_{B G_{l}}=\mathbf{R}_{\varphi, \theta, \epsilon, \delta}\left[\begin{array}{c}
x_{G l} \\
0 \\
\lambda_{f}+z_{G l}
\end{array}\right]
$$

By differentiating, the relative linear and angular velocity vectors are:

$$
\begin{aligned}
\dot{\boldsymbol{r}}_{v G_{l}} & =\dot{\boldsymbol{r}}_{v B}+\dot{\boldsymbol{r}}_{B G_{l}}=\dot{\boldsymbol{r}}_{v B}+\boldsymbol{\omega}_{\varphi, \theta, \delta} \times \boldsymbol{r}_{B G_{l}}+\dot{\lambda}_{f} \boldsymbol{k}_{\delta} \\
\boldsymbol{\omega}_{v G_{l}} & =\boldsymbol{\omega}_{v G_{r}}+\boldsymbol{\omega}_{\delta}
\end{aligned}
$$

where the coordinates of the unit vector $k_{\delta}$ in $\Re_{v}$ are $\boldsymbol{k}_{\delta}=\mathbf{R}_{\varphi, \theta, \epsilon, \delta}[0,0,1]^{T}$.

\subsubsection{Swing arm body}

The swing arm body has 3 DoF w.r.t $\Re_{v}$ which represents the roll, swing rotations and vertical displacement. Then, its center of mass position vector is:

$$
\boldsymbol{r}_{v G_{s}}=\boldsymbol{r}_{v P}+\boldsymbol{r}_{P G_{s}}
$$

in which the coordinates of $\boldsymbol{r}_{P G_{s}}$ in the reference frame $\Re_{v}$ are calculated using the following expression:

$$
\boldsymbol{r}_{P G_{s}}=\mathbf{R}_{\varphi, \mu}\left[\begin{array}{c}
x_{G s} \\
0 \\
z_{G s}
\end{array}\right]
$$

By differentiating, the relative linear and angular velocity vectors are:

$$
\begin{aligned}
\dot{\boldsymbol{r}}_{v G_{s}} & =\dot{\boldsymbol{r}}_{v P}+\dot{\boldsymbol{r}}_{P G_{s}}=\dot{\boldsymbol{r}}_{v P}+\boldsymbol{\omega}_{\varphi, \mu} \times \boldsymbol{r}_{P G_{s}} \\
\boldsymbol{\omega}_{v G_{s}} & =\boldsymbol{\omega}_{\varphi, \mu}=\boldsymbol{\omega}_{\varphi}+\boldsymbol{\omega}_{\mu}
\end{aligned}
$$

where $\boldsymbol{\omega}_{\mu}=\mathbf{R}_{\varphi, \mu}[0, \dot{\mu}, 0]^{T}$. 


\subsubsection{Rear-wheel bodies}

The rear-wheel body has three independent DoF w.r.t $\Re_{v}$ including roll rotation, vertical displacement and the tire spin rotation around wheel spin axis $\boldsymbol{j}_{\zeta, r}$. It follows that:

$$
\boldsymbol{\omega}_{v R_{r}}=\boldsymbol{\omega}_{\varphi}+\boldsymbol{\omega}_{\zeta, r}
$$

where the components of the angular velocity are $\boldsymbol{\omega}_{\zeta, r}=\mathbf{R}_{\varphi}\left[0, \dot{\zeta}_{r}, 0\right]$. On the other hand, the position of the rear wheel center of mass is:

$$
\boldsymbol{r}_{v R_{r}}=\boldsymbol{r}_{v P}+\boldsymbol{r}_{P R_{r}}
$$

where the coordinates of the vector $\boldsymbol{r}_{P R_{r}}$ are computed by:

$$
\boldsymbol{r}_{P R_{r}}=\mathbf{R}_{\varphi, \mu}\left[\begin{array}{c}
x_{R r} \\
0 \\
z_{R r}
\end{array}\right]
$$

By differentiating, the relative linear velocity vector is:

$$
\dot{\boldsymbol{r}}_{v R_{r}}=\dot{\boldsymbol{r}}_{v P}+\dot{\boldsymbol{r}}_{P R_{r}}=\dot{\boldsymbol{r}}_{v P}+\boldsymbol{\omega}_{\varphi, \mu} \times \boldsymbol{r}_{P R_{r}}
$$

\subsubsection{Front-wheel body}

The front-wheel body has one independent DoF w.r.t $G_{l}$ which represents the tire spin rotation around wheel spin axis $\boldsymbol{j}_{\zeta, f}$, consequently:

$$
\boldsymbol{\omega}_{v R_{f}}=\boldsymbol{\omega}_{v G_{l}}+\boldsymbol{\omega}_{\zeta_{f}}
$$

where $\boldsymbol{\omega}_{\zeta_{f}}=\mathbf{R}_{\varphi, \theta, \epsilon, \delta}\left[0, \dot{\zeta}_{f}, 0\right]^{T}$. In the same way as the rear wheel, the position of the front wheel center of mass is:

$$
\boldsymbol{r}_{v R_{f}}=\boldsymbol{r}_{v B}+\boldsymbol{r}_{B R_{f}}
$$

in which the coordinates of the vector $\boldsymbol{r}_{B R_{f}}$ are:

$$
\boldsymbol{r}_{B R_{f}}=\mathbf{R}_{\varphi, \theta, \epsilon, \delta}\left[\begin{array}{c}
x_{R f} \\
0 \\
\lambda_{f}+z_{R f}
\end{array}\right]
$$


By differentiating, the relative linear velocity vector is:

$$
\dot{\boldsymbol{r}}_{v R_{f}}=\dot{\boldsymbol{r}}_{v B}+\dot{\boldsymbol{r}}_{B R_{f}}=\dot{\boldsymbol{r}}_{v B}+\boldsymbol{\omega}_{\varphi, \theta, \delta} \times \boldsymbol{r}_{B R_{f}}+\dot{\lambda}_{f} \boldsymbol{k}_{\delta}
$$

At this level, the whole motorcycle kinematics is defined. Matrix mass $\mathbf{M}$ and the residual generalized efforts $\boldsymbol{Q}_{R}$ are fully defined. In the next section, we look for the development of the generalized efforts vector associated to external applied efforts.

\section{Vector of Generalized Efforts}

The motorcycle system is subject to various forces and torques elements. One enumerates gravity forces, suspensions forces due to springs and shock-absorbers, tire forces and torques at the tire/road contact point, rider steering torque and steer damper torque applied on motorcycle's handlebar, rear and front brake torques and finally the driving torque applied on the rear wheel. In this work, the rider is considered to be a rigid body attached to the rear assembly $G_{r}$ where its lean movement is ignored.

To express the generalized efforts vector $\boldsymbol{Q}_{a}$ associated to external applied efforts, one can make use of equation (12). This method may be tedious, especially when the applied efforts are numerous. So, it is most convenient to find the virtual power done by each effort and hence its associated contribution.

\subsection{Gravity force}

The contribution of this force in $\boldsymbol{Q}_{a}$ is directly computed from equation (12):

$$
\boldsymbol{Q}_{a, g}=\sum_{i}\left(\frac{\partial \boldsymbol{v}_{o i}}{\partial \boldsymbol{\vartheta}}\right)^{T}\left[\begin{array}{c}
0 \\
0 \\
-m_{i} g
\end{array}\right]
$$

for $i \in\left(G_{r}, G_{f}, G_{l}, G_{s}, R_{f}, R_{r}\right)$.

\subsection{Rider torque}

The rider's torque $\tau_{r}$ applied on the motorcycle's handlebar is considered to be an important input for the vehicle riding. This torque is applied around the steering axis, which constitutes the interconnection link between the rear body $G_{r}$ and the front upper body $G_{f}$. The contribution of this torque is defined by:

$$
\boldsymbol{Q}_{a, h}=\left(\frac{\partial \dot{\delta}}{\partial \boldsymbol{\vartheta}}\right)^{T}\left[\begin{array}{c}
0 \\
0 \\
\tau_{r}-C_{\delta} \dot{\delta}
\end{array}\right]=\left[\begin{array}{c}
\mathbf{0}_{6} \\
\tau_{r}-C_{\delta} \dot{\delta} \\
\mathbf{0}_{4}
\end{array}\right]
$$

where $-C_{\delta} \dot{\delta}$ is the steer damping torque.

In [25], a more detailed rider-motorcycle interaction is described by taking into account the rider's upper torso leaning motion and the connection of his/her arms with the handlebars. 


\subsection{Propulsion and braking torque}

For the front-wheel, the braking force acts between the wheel knuckle and the front lower body which contains the braking disk. For the rear wheel, the braking torque is applied between the rear-wheel body and the swing arm assembly where the engine propulsion torque is applied around the rear-wheel spin axis, hence:

$$
\begin{aligned}
\boldsymbol{Q}_{a, p b} & =\left(\frac{\partial \dot{\zeta}_{f}}{\partial \boldsymbol{\vartheta}}\right)^{T}\left[\begin{array}{c}
0 \\
\tau_{B, f} \\
0
\end{array}\right]+\left(\frac{\partial \dot{\zeta}_{r}}{\partial \boldsymbol{\vartheta}}\right)^{T}\left[\begin{array}{c}
0 \\
\tau_{D}+\tau_{B, r} \\
0
\end{array}\right]+\left(\frac{\partial \dot{\mu}}{\partial \boldsymbol{\vartheta}}\right)^{T}\left[\begin{array}{c}
0 \\
-\tau_{B, r} \\
0
\end{array}\right] \\
& =\left[\begin{array}{c}
\mathbf{0}_{8} \\
-\tau_{B, r} \\
\tau_{B, f} \\
\tau_{D}+\tau_{B, r}
\end{array}\right]
\end{aligned}
$$

\subsection{Front and rear suspension}

The front suspension force $F_{s, f}$ is applied between the front upper and the front lower bodies. The contribution of this force in $\boldsymbol{Q}_{a}$ can be expressed by using the virtual power principle, therefore:

$$
\boldsymbol{Q}_{a, s f}=\left(\frac{\partial \dot{\lambda}_{f}}{\partial \boldsymbol{\vartheta}}\right)^{T} F_{s, f}=\left[\begin{array}{c}
\mathbf{0}_{7} \\
F_{s, f} \\
\mathbf{0}_{3}
\end{array}\right]
$$

In the same way, the contribution of the rear suspension can be computed as following:

$$
\boldsymbol{Q}_{a, s r}=\left(\frac{\partial \dot{\lambda}_{r}}{\partial \boldsymbol{\vartheta}}\right)^{T} F_{s, r}
$$

where $\lambda_{r}$ is the rear suspension travel given by $\lambda_{r}^{2}=\boldsymbol{r}_{S_{l} S_{u}} \cdot \boldsymbol{r}_{S_{l} S_{u}}$. By differentiating, the suspension travel rate $\dot{\lambda}_{r}$ is deduced and written by the following equation:

$$
\dot{\lambda}_{r}=\boldsymbol{e}_{S_{l} S_{u}} \cdot\left\{\boldsymbol{\omega}_{\varphi, \theta} \times \boldsymbol{r}_{P S_{u}}-\boldsymbol{\omega}_{\varphi, \mu} \times \boldsymbol{r}_{P S_{l}}\right\}
$$



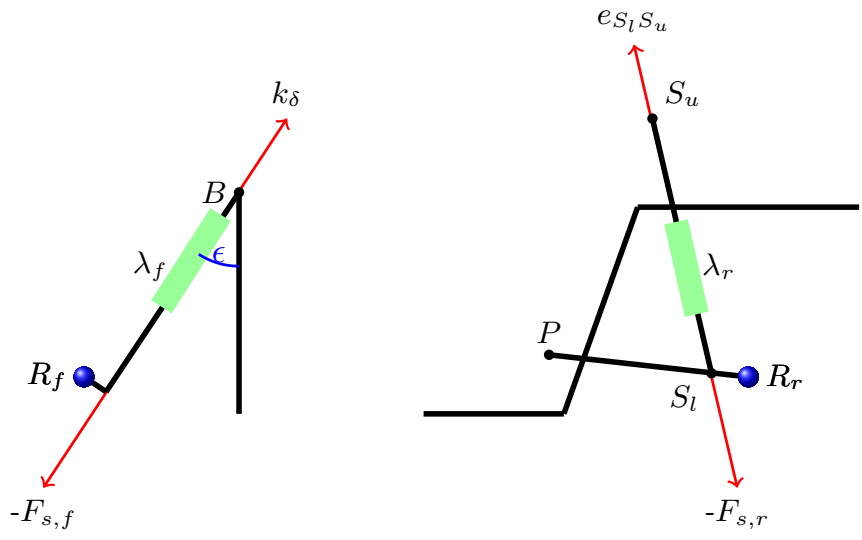

Figure 4: Suspensions configuration, (a) front, (b) rear

where $e_{S_{l} S_{u}}$ is the rear suspension unit vector. Consequently, the contribution of the rear suspension in the total generalized effort is given by:

$$
\boldsymbol{Q}_{a, s r}=\left[\begin{array}{c}
\mathbf{0}_{5} \\
\boldsymbol{e}_{S_{l} S_{u}} \cdot\left(\boldsymbol{j}_{\theta} \times \boldsymbol{r}_{P S_{u}}\right) \\
\mathbf{0}_{2} \\
-\boldsymbol{e}_{S_{l} S_{u}} \cdot\left(\boldsymbol{j}_{\mu} \times \boldsymbol{r}_{P S_{l}}\right) \\
\mathbf{0}_{2}
\end{array}\right] F_{s, r}
$$

where the coordinates of the unit vectors $\boldsymbol{j}_{\theta}$ and $\boldsymbol{j}_{\mu}$ in $\Re_{v}$ are respectively equal to $\mathbf{R}_{\varphi}[0,1,0]^{T}$.

Equations (42-48) show the contribution in $\boldsymbol{Q}_{a}$ of each external force/moment applied on the motorcycle vehicle. In the next section, the tire-road interaction is exposed and its contribution is demonstrated.

\section{Tires' kinematics and dynamics}

Tire-road interaction is the most important phenomena that characterize ground vehicles. Due to this importance, several works are undertaken leading to a mathematical description of friction forces which arises from tire and carcass deformation $[15,26]$.

In the present paper, the motorcycle's wheels are considered as rigid, thin disks where the road/tire contact is dot shaped at point $c$. At this point, a system of longitudinal, lateral and vertical forces is implemented by using an empirical tire model type named Pacejka model [27] with respect to kinematic slip quantities and the normal load. Moreover, carcass deformations are not taken into account but reproduced by introducing the tire relaxation equations [20].

In following, we expose the tire kinematics to define slip variables required while computing the tire/road contact forces and hence, deduce their contribution in the generalized effort vector $\boldsymbol{Q}_{a}$. 


\subsection{Tires kinematics}

To describe the tire motion, a new reference frame $\Re_{T}\left(c, \boldsymbol{i}_{T}, \boldsymbol{j}_{T}, \boldsymbol{k}_{T}\right)$ is introduced at the contact point $c$ of each wheel's tire. $\boldsymbol{k}_{T}$ is the normal vector to the road surface. Vector $\boldsymbol{i}_{T}$ is obtained by the cross product $\boldsymbol{j}_{\zeta} \times \boldsymbol{k}_{T}$ and $\boldsymbol{j}_{T}$ completes the reference axis.

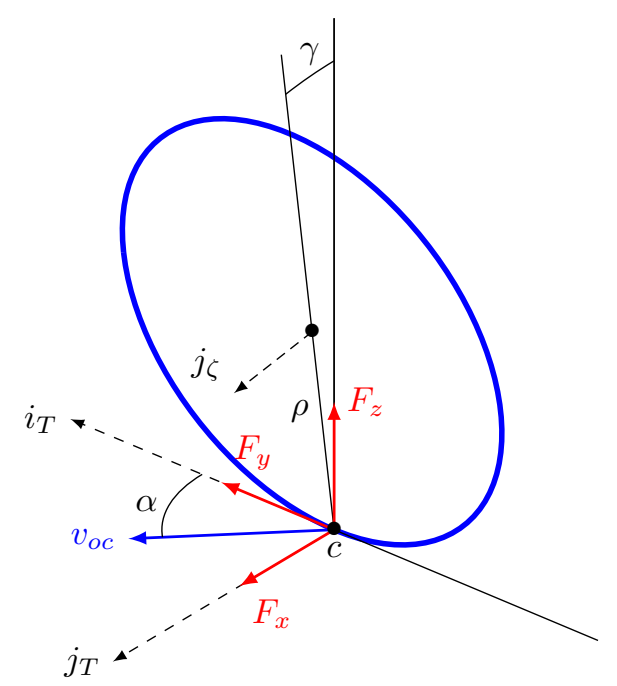

Figure 5: Tire reference frame

As shown in Figure 5, the camber angle is an important variable in the motorcycle safety studies [28]. This variable is expressed by the following scalar product:

$$
\sin \gamma=\boldsymbol{j}_{\zeta} \cdot \boldsymbol{k}_{T}
$$

Besides, the position of the rear and front road/tire contact point $c$ w.r.t the movable frame reference $\Re_{v}$ is given by:

$$
\begin{aligned}
& \boldsymbol{r}_{v c_{r}}=\boldsymbol{r}_{v R_{r}}+\boldsymbol{r}_{R_{r} c_{r}} \\
& \boldsymbol{r}_{v c_{f}}=\boldsymbol{r}_{v R_{f}}+\boldsymbol{r}_{R_{f} c_{f}}
\end{aligned}
$$

in which the coordinates of $\boldsymbol{r}_{R_{r} c_{r}}$ and $\boldsymbol{r}_{R_{f} c_{f}}$ in $\Re_{v}$ are respectively given by:

$$
\boldsymbol{r}_{R_{r} c_{r}}=\mathbf{R}_{\varphi}\left[\begin{array}{c}
0 \\
0 \\
-\rho_{r}
\end{array}\right] \quad \boldsymbol{r}_{R_{f} c_{f}}=\mathbf{R}_{\varphi, \theta, \epsilon, \delta}\left[\begin{array}{c}
-\rho_{f} \sin \epsilon \\
0 \\
\rho_{f} \cos \epsilon
\end{array}\right]
$$

By differentiating, the relative linear velocity vector of each contact point can be deduced as: 


$$
\begin{aligned}
\dot{\boldsymbol{r}}_{v c_{r}} & =\dot{\boldsymbol{r}}_{v R_{r}}+\boldsymbol{\omega}_{\varphi} \times \boldsymbol{r}_{R_{r} c_{r}} \\
\dot{\boldsymbol{r}}_{v c_{f}} & =\dot{\boldsymbol{r}}_{v R_{f}}+\boldsymbol{\omega}_{v G_{f}} \times \boldsymbol{r}_{R_{f} c_{f}}
\end{aligned}
$$

By using equation (2), the contact point velocity vector is computed and the slip variables are defined by the following equations:

$$
\begin{aligned}
\alpha_{r} & =-\operatorname{atan}\left(\frac{\boldsymbol{j}_{v} \cdot \boldsymbol{v}_{o c_{r}}}{\boldsymbol{i}_{v} \cdot \boldsymbol{v}_{o c_{r}}}\right) \\
\alpha_{f} & =-\operatorname{atan}\left(\frac{\boldsymbol{j}_{v} \cdot \boldsymbol{v}_{o c_{f}}}{\boldsymbol{i}_{v} \cdot \boldsymbol{v}_{o c_{f}}}\right)+\delta \cos \epsilon \\
\kappa_{i} & =-\frac{V_{c, i}-r_{D, i} \dot{\zeta}_{i}}{\max \left(V_{c, i}, r_{D, i} \dot{\zeta}_{i}\right)}
\end{aligned}
$$

where, $V_{c, i}=\left\|\boldsymbol{v}_{o c_{i}}\right\|$ is the contact point speed, $r_{D, i}$ is the wheel's dynamic radius and $i=r, f$.

Finally, knowing that the contact point $c_{i}$ must always belong to the road surface then, the vertical deformations $\delta_{z, i}$ of the wheels' tire can be derived by:

$$
\delta_{z, i}=\boldsymbol{k}_{T} \cdot \boldsymbol{r}_{v c_{i}}
$$

These deformations help to determine the normal load necessary to maintain contact with the road plane [29].

\subsection{Tires force/moment system}

Once the tire's kinematics is established, the equivalent tire forces/moments wrench at the center of each wheel is given by the following expressions:

$$
\begin{aligned}
\boldsymbol{F}_{T} & =F_{x} \boldsymbol{i}_{T}+F_{y} \boldsymbol{j}_{T}+F_{z} \boldsymbol{k}_{T} \\
\boldsymbol{M}_{T} & =M_{x} \boldsymbol{i}_{T}+M_{y} \boldsymbol{j}_{T}+M_{z} \boldsymbol{k}_{T}+\boldsymbol{F}_{T} \times \boldsymbol{r}_{c R}
\end{aligned}
$$

where $F_{x}=F_{x}(\kappa)$ is the longitudinal force, $F_{y}=F_{y}(\alpha, \gamma)$ is the lateral force and $F_{z}=F_{z}\left(\delta_{z}\right)$ is the vertical force. $M_{x}$ is the tire torque about $\boldsymbol{i}_{T}, M_{y}$ is the rolling resistance torque and $M_{z}=M_{z}(\alpha, \gamma)$ is the alignment torque. These forces and torques are obtained from equation (53) for the longitudinal slip $\kappa$, the lateral slip $\alpha$ and from equation (54) for the vertical tire deformation $\delta_{z}$. Afterwards, the contribution of the tire/road contact forces/moments in the generalized efforts vector is given by:

$$
\boldsymbol{Q}_{a, T}=\sum_{i=f, r}\left\{\left(\frac{\partial \boldsymbol{v}_{o R_{i}}}{\partial \boldsymbol{\vartheta}}\right)^{T} \boldsymbol{F}_{T, i}+\left(\frac{\partial \boldsymbol{\omega}_{o R_{i}}}{\partial \boldsymbol{\vartheta}}\right)^{T} \boldsymbol{M}_{T, i}\right\}
$$




\section{Motorcycle model control}

To simulate the motorcycle model, a convenient control is to be synthesized to track a reference longitudinal speed profile and to stabilize the lateral dynamics around trim trajectories. This is a hard task since the roll DoF is unstable and, for some aggressive driving maneuvers conditions, system nonlinearities cannot be neglected. In addition, it is impossible to exploit the whole nonlinear model to develop a suitable controller, even by using a nonlinear control method. For these reasons, a linearized version of the dynamics model representing small motions in the neighborhood of the straight motion is considered.

In this paper, the speed and the roll controllers are based on Lyapunov method [30]. This technique avoids the gain-scheduling, necessary for the linear method control to consider longitudinal speed variations. For each mode, longitudinal and lateral, the controller acts on the speed error to produce a torque to be applied on the rear wheel and on the roll angle error to generate a rider steering torque to be applied on the motorcycle's handlebar.

\subsection{Longitudinal control}

Consider the following longitudinal simplified model:

$$
\begin{aligned}
\sum m_{i}\left(\dot{v}_{x}-v_{y} \dot{\psi}\right) & =F_{x, f}+F_{x, r}-F_{y, f} \sin \delta \\
i_{y, f} \ddot{\zeta}_{f} & =\tau_{b, f}-\rho_{f}\left(F_{x, f}-F_{y, f} \sin \delta\right) \\
i_{y, r} \ddot{\zeta}_{r} & =\tau_{D}+\tau_{b, r}-\rho_{r} F_{x, r}
\end{aligned}
$$

With the assumption of zero longitudinal slip, it goes that $v_{x}=\rho_{r} \dot{\zeta}_{r}=\rho_{f} \dot{\zeta}_{f}$ and by replacing in equation (56) the simplified longitudinal mode becomes:

$$
m_{e q} \dot{v}_{x}=\frac{\tau_{b, f}}{\rho_{f}}+\frac{\tau_{D}+\tau_{b, r}}{\rho_{r}}+\sum m_{i} v_{y} \dot{\psi}
$$

where $m_{e q}=\sum m_{i}+i_{y, f} / \rho_{f}^{2}+i_{y, r} / \rho_{r}^{2}$ is the equivalent mass. Let us introduce the Lyapunov function $V=m_{e q} e^{2} / 2$, where $e=v_{x}-v_{x, r e f}$ is the speed tracking error, its derivative is given by:

$$
\dot{V}=m_{e q} e \dot{e}=e\left\{\frac{\tau_{b, f}}{\rho_{f}}+\frac{\tau_{D}+\tau_{b, r}}{\rho_{r}}+\sum m_{i} v_{y} \dot{\psi}-m_{e q} \dot{v}_{x, r e f}\right\}
$$

In order to the Lyapunov function derivative to be negative, the propulsion torque $\tau_{D}$ must satisfy:

$$
\tau_{D}=\rho_{r}\left\{-k_{x} e_{x}-\frac{\tau_{b, f}}{\rho_{f}}-\sum m_{i} v_{y} \dot{\psi}+m_{e q} \dot{v}_{x, r e f}\right\}-\tau_{b, r}
$$


where $k_{x}$ is a positive constant. With this input control, the Lyapunov function derivative is always negative $\left(\dot{V}=-k_{x} e^{2}\right)$ and hence the system is also, always stable.

Remark With the control of equation (59), the error dynamics becomes $\dot{e}=-k_{x} e$, then, $k_{x}$ is just a proportional coefficient which is tuned until the desired tracking error level is achieved. However, a high value introduces an oscillatory behavior while stability remains preserved. 

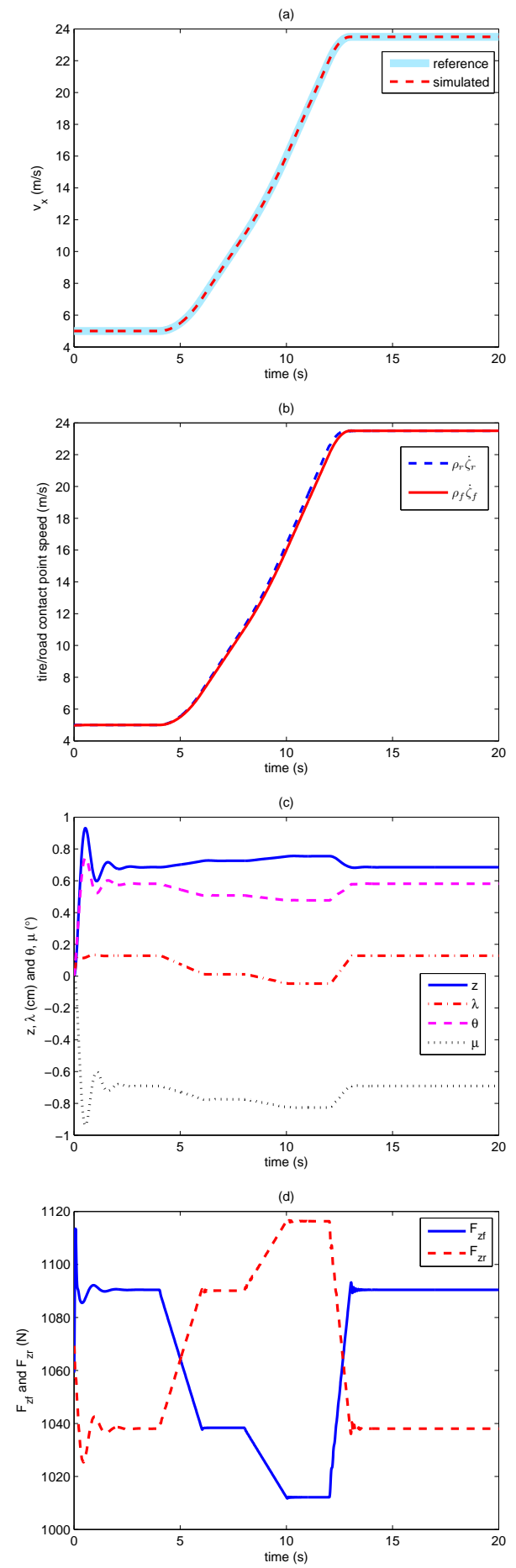

Figure 6: Speed profile tracking 

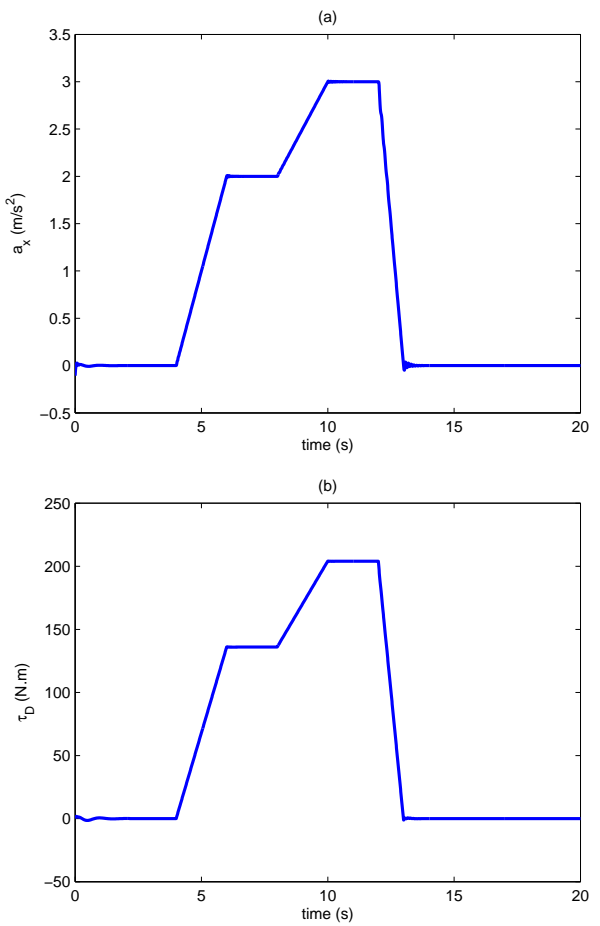

Figure 7: Longitudinal acceleration and requested propulsion torque

The following simulations are carried-out using the developed nonlinear model and the controller of equation (59). Starting from $5 \mathrm{~ms}^{-1}$, the desired speed profile consists of two main phases, an acceleration phase to reach $23 \mathrm{~m} / \mathrm{s}^{-1}$ followed by a deceleration phase to keep a constant speed (see Figure 6-a). In Figure 6-b, the tire/road contact point speed is shown to give an overview of the longitudinal slip where Figure 6-c presents the simulation results of the in-plane mode variables (vertical displacement, front suspension travel, pitch and swing angles). Figure 6-d, describes the evolution of the nominal load applied on each tire w.r.t to speed variations (and so longitudinal acceleration). Lastly, requested propulsion torque at the rear-wheel and the resulting acceleration are sketched in Figure 7.

The second simulation aims to highlight the motorcycle behavior when subjected to a braking torques applied separately on the front and the rear wheel. Here in, it is not question to apply a particular braking model such as the ABS system. It will be shown that when applying a braking torque, significant load transfers take place from the rear wheel to the front one. If suspensions are assumed to be rigid, an approximation of the vertical load on each wheel when braking [31] is given by: 


$$
\begin{aligned}
F_{z, f} & =\frac{\sum m g\left(x_{G r}-x_{R r}\right)+F_{B} z_{G r}}{p} \\
F_{z, r} & =\frac{\sum m g\left(p-x_{G r}-x_{R r}\right)-F_{B} z_{G r}}{p}
\end{aligned}
$$

where, $p$ is the motorcycle wheelbase and $F_{B}$ is the total braking force at the tire/road contact. Based on these two equations, a limit braking force $F_{B \text {, lim }}$ which causes the rear wheel lift can be computed.

Figure 8 represents the rear and the front brake profile applied separately on each wheel. In the first scenario, a rear braking torque with a maximum value of $-600 \mathrm{~N} . \mathrm{m}$ is applied between 4-7 seconds. The motorcycle dynamics behavior is demonstrated in Figure 9. From an initial forward speed of $20 \mathrm{~m} / \mathrm{s}$, the motorcycle brakes hardly up to $2 \mathrm{~m} / \mathrm{s}$ with a deceleration of $0.4 \mathrm{~g}$. In that case, the vertical position $z$ of point $P$ is lowered which introduces a clockwise and hence a negative pitch rotation $\theta$. The same vertical movement produces an anti-clockwise and hence a positive swing rotation $\mu$. It is clear that the rear wheel does not lift due to the applied vertical force $(600 \mathrm{~N}$ which is equivalent to a payload of $60 \mathrm{~kg}$. See Figure 9-d).

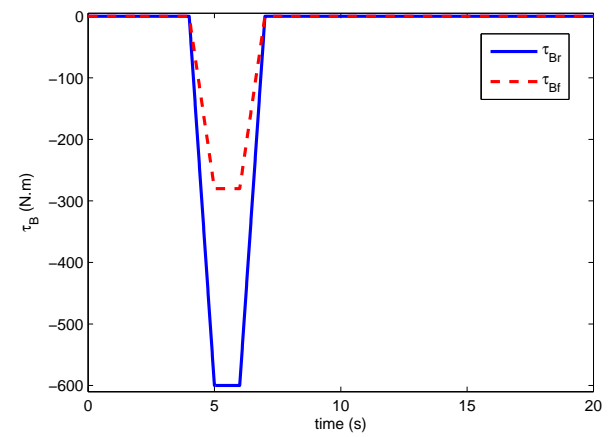

Figure 8: Rear and front brake torque profiles

In the second scenario, the same torque profile as before is applied but with minus a half amplitude $(-280 \mathrm{Nm})$. The motorcycle dynamics behavior is shown in Figure 10. Initially the motorcycle was traveling at $20 \mathrm{~m} / \mathrm{s}$ and brakes up to $12 \mathrm{~m} / \mathrm{s}$ with a deceleration of $0.4 \mathrm{~g}$. A first conclusion concerns the amplitude of the applied torque which is not sufficient to stop the motorcycle. In addition, such front brake can easily create the limit braking force $F_{B \text {, lim }}$ and hence causes the loss of the rear wheel contact with the ground. Indeed, the vertical force at the rear wheel is about $100 \mathrm{~N}$ (a payload of only 10kg. See Figure 10-d). With the same justification, the vertical position $z$ of point $P$ goes up which introduces an anti-clockwise and hence a positive pitch rotation $\theta$ and a clockwise and hence a negative swing rotation $\mu$. 

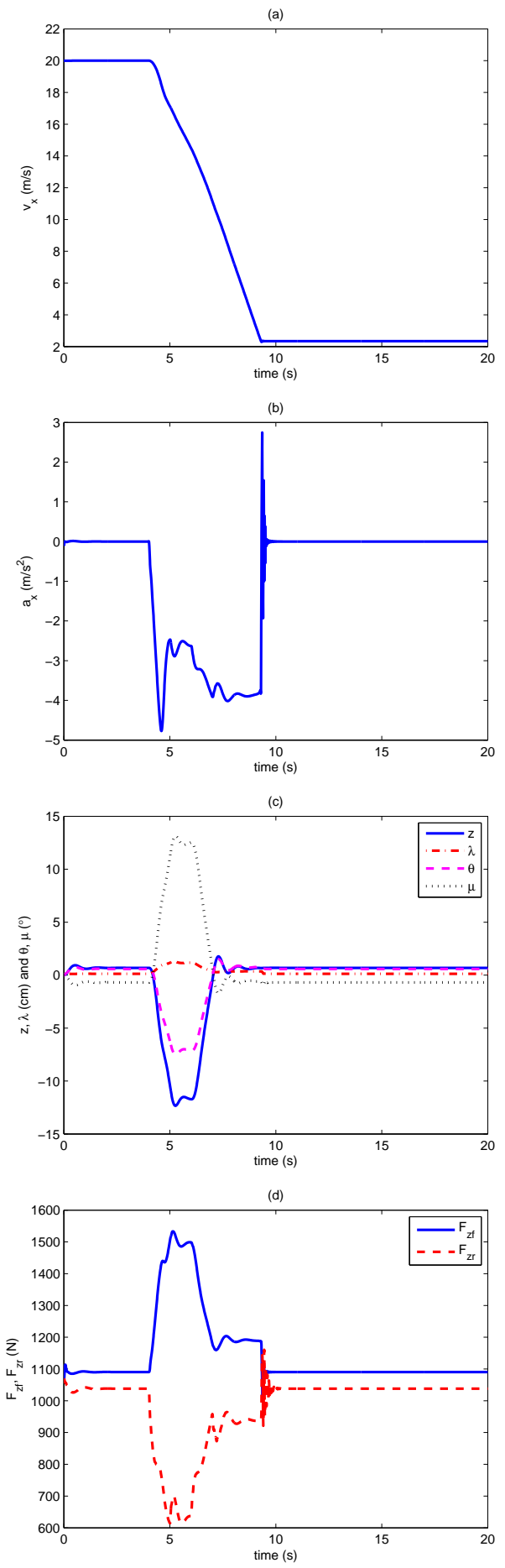

Figure 9: Simulation results according to only hard rear brake torque 

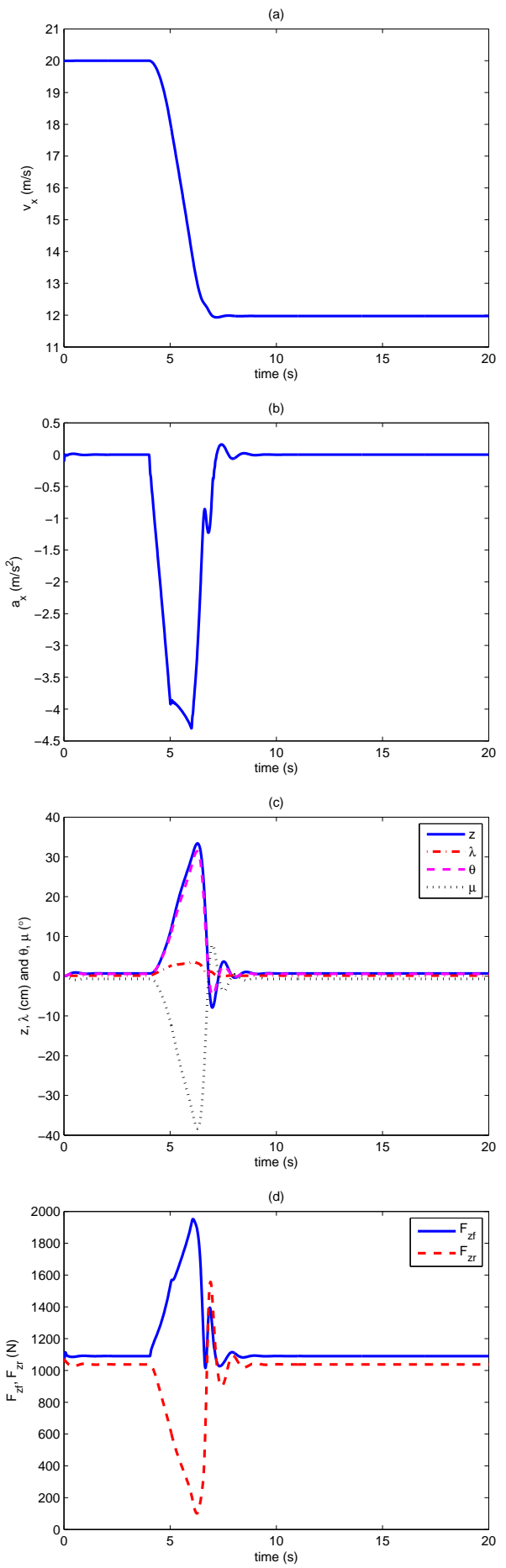

Figure 10: Simulation results according to only hard front brake torque 
As is recognized in literature, this simulation presents the braking efficiency where an optimal braking must be split between the two wheels with more brake ratio at the front wheel (Figure 11).
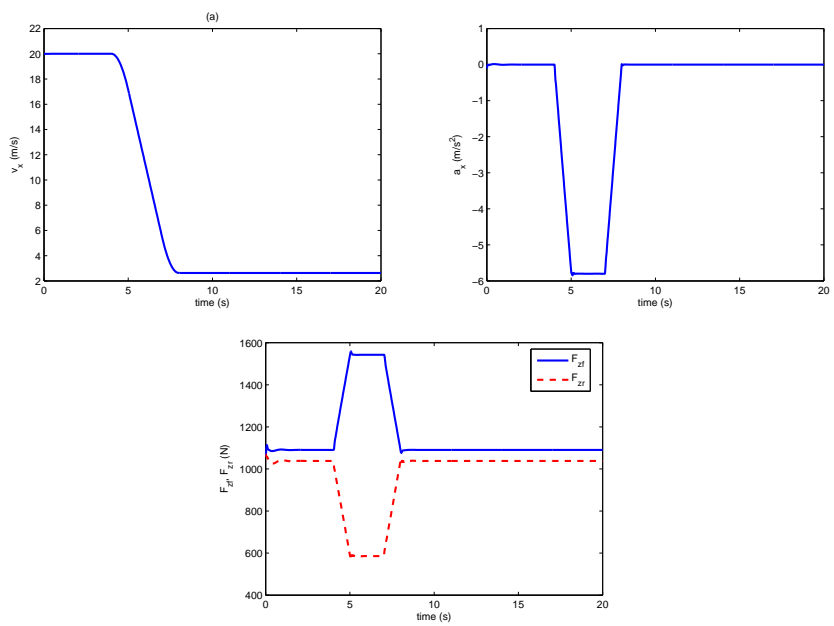

Figure 11: Simulation results according to $70 \%$ front brake torque and $30 \%$ rear brake torque of previous torques

\subsection{Cornering maneuver control}

As the longitudinal mode, a linearized version of the lateral dynamics model representing small motions in the neighborhood of the straight motion is derived which leads to the following linear state-space representation:

$$
\dot{\boldsymbol{x}}=\mathbf{A}_{L} \boldsymbol{x}+\boldsymbol{B} \tau_{r}
$$

where $\boldsymbol{x}=\left[v_{y}, \dot{\psi}, \dot{\varphi}, \dot{\delta}, \varphi, \delta\right]^{T}$ is the state vector and $\tau_{r}$ is the rider torque input. In Figure 12, the stability of the uncontrolled motorcycle model of equation (61) is presented. This eigenvalues plot shows the existence of stable modes (in $\circ$ symbol) and three instability modes, the first concerns the steering motion which appears from $8 \mathrm{~m} / \mathrm{s}$ (in $\star$ symbol), the second concerns the roll motion at very low speed until $7 \mathrm{~m} / \mathrm{s}$ (in $\nabla$ symbol) and finally, a coupled steering-roll instable motion generated at very low speed (in + symbol). In [11], these instability modes are named capsize, weave and wobble. 


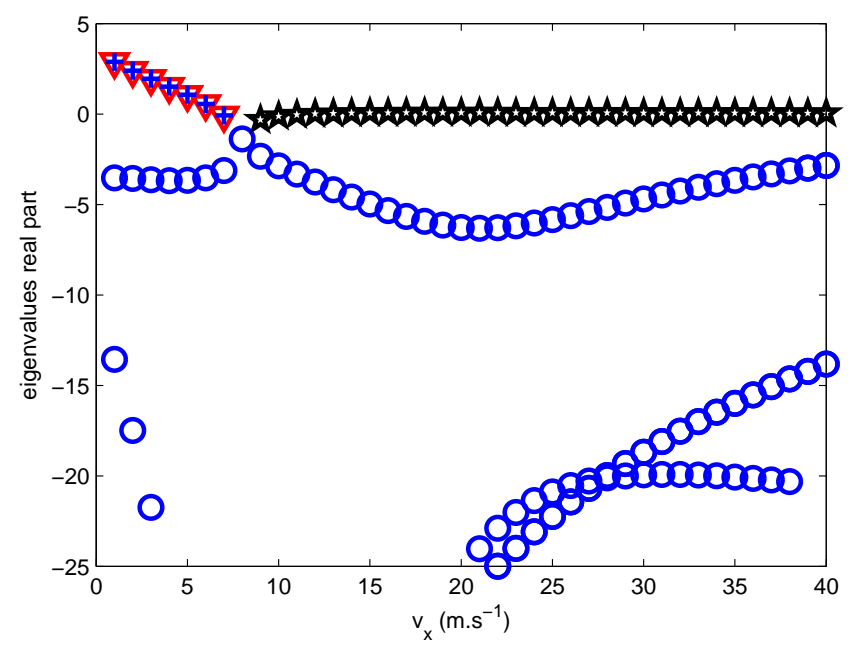

Figure 12: Eigenvalues real part of the uncontrolled linearized motorcycle model w.r.t the forward speed

In order to stabilize the motorcycle, a control scheme is to be synthesized to track a reference roll angle. In its simplest form, consider the Lyapunov function $V=e^{2} / 2$, where $e=\varphi-\varphi_{\text {ref }}$ is the roll tracking error. By differentiation, the roll error dynamics becomes:

$$
\begin{aligned}
& \dot{e}=\dot{\varphi}-\dot{\varphi}_{\text {ref }} \\
& \ddot{\varphi}=\mathbf{A}_{L,(3,:)} \boldsymbol{x}+\boldsymbol{B}_{3} \tau_{r}
\end{aligned}
$$

where $\boldsymbol{B}_{3}$ and $\mathbf{A}_{L,(3,:)}$ are the third line of vector $\boldsymbol{B}$ and matrix $\mathbf{A}_{L}$. From equation (62), it can be seen that $\dot{\varphi}$ acts as a virtual input to the roll error dynamics. By using backstepping technique [32], the required rider torque to track a reference roll angle is given by:

$$
\begin{aligned}
\boldsymbol{B}_{3} \tau_{r} & =-k_{z} z-\mathbf{A}_{L,(3,:)} \boldsymbol{x}+\dot{\beta} \\
\beta & =-k_{\varphi} e+\dot{\varphi}_{r e f}
\end{aligned}
$$

where $z=\dot{\varphi}-\beta$ is the error between the real roll rate and the virtual input $\beta$. 


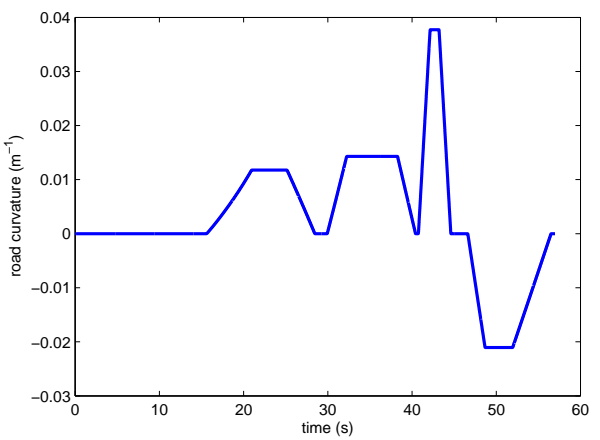

Figure 13: Road curvature

The following simulation are carried-out using the nonlinear model and the controllers of equations $(59,63)$ where Figure 13 shows the road curvature. From this curvature, the reference roll profile is calculated. In Figure 14-a, the performance of backstepping controller in reference tracking is described. Obviously, the linear modelbased control of equation (63) is able to stabilize the nonlinear dynamics. However, for an aggressive cornering maneuver where the system nonlinearities are important, the stability of the motorcycle is not guaranteed. 

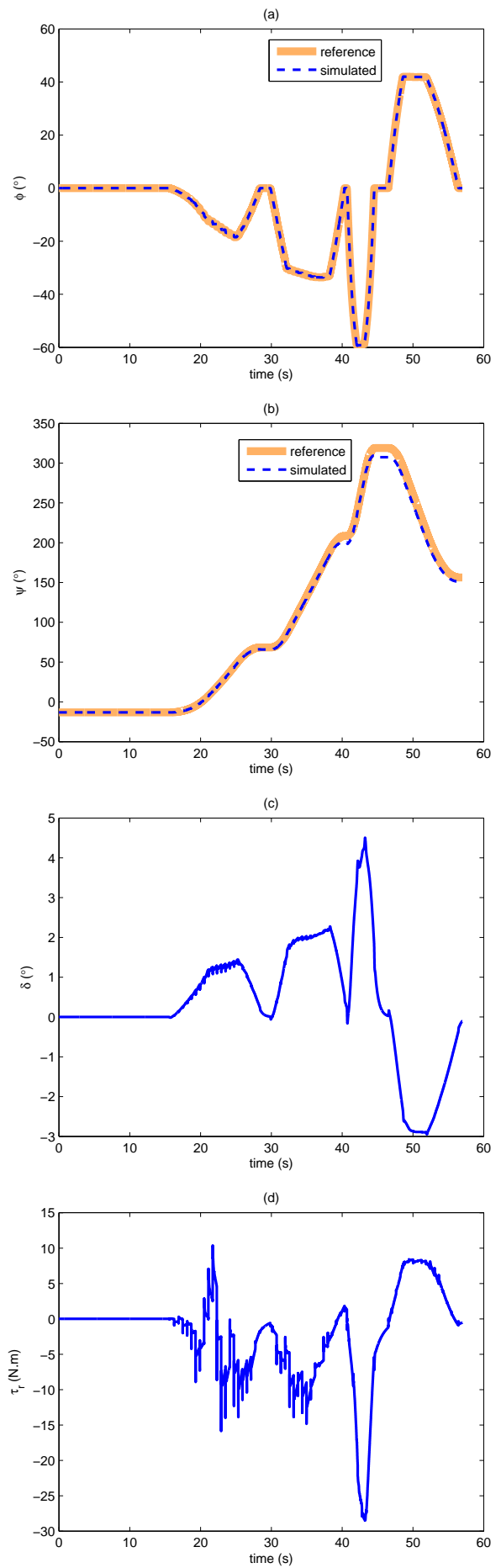

Figure 14: Simulation results for roll profile tracking 26 
Otherwise, from figures 14-(a,b,c), the main features of the proposed dynamic model are illustrated mainly the counter-steer phenomena. Indeed, for a given applied torque $\tau_{r}$, the vehicle leans in the same direction as torque sign whereas the vehicle is steered in the opposite direction. Furthermore, steering angle and curvature have approximately the same plot. Finally, the requested rider torque at the motorcycle's handlebar is illustrated in Figure 14-d.

\section{Conclusion}

Based on the virtual power principle, called also Jourdain's principle, a middle complexity motorcycle dynamics model is proposed. The multi-body model has $11 \mathrm{DoF}$ and includes the main features of motorcycle subsystems. This approach is chosen for its simplicity of synthesis and its fine analytical derivation with an acceptable calculation load, in particular, when the equations of motion are developed by using generalized coordinates approach. This model exhibits many of interesting behaviors of a real motorcycle such non-minimum phase steering response and load transfer resulting from acceleration and braking.

Besides, a virtual rider is synthesized for longitudinal and cornering modes respectively by using Lyapunov theory. This technique allows the calculation of speed and roll controllers which are valid for a wide range of forward speeds and hence, avoids controller scheduling.

From Figure 14.b, it is clear that using only roll stabilization cannot lead to a good trajectory tracking. For this, additional cost functions must be included to minimize the relative yaw angle and lateral deviation errors of the motorcycle from the road center line. These issues will be the scope of future works. Moreover, as perspective, experimental validation of the proposed motorcycle model and the simulation of more limit riding maneuvers are expected.

\section{References}

[1] from www.preventionroutiere.asso.fr

[2] H. Arioui, L. Nehaoua, S. Hima, N. Sguy and S. Espi, Mechatronics, Design, and Modeling of a Motorcycle Riding Simulator, IEEE/ASME Trans. on Mechatronics, 15(5), (2010), pp. 805-818.

[3] ADvAnced Multi-body Simulation, http ://www.mscsoftware.com

[4] http :// www.mathworks.fr

[5] M. Tanelli, F. Schiavo, S.M. Savaresi and G. Ferretti, Object-Oriented Multibody Motorcycle Modelling for Control Systems Prototyping, Proc. IEEE CACSD, Munich, Germany, 2006.

[6] R. Lot and M. Da Lio, A Symbolic Approach for Automatic Generation of the Equations of Motion of Multibody Systems, j. of MSD, 12(2000), pp. 147-172. 
[7] F.J.W. Whipple, Stability of the motion of a bicycle, Quarterly J. of Pure and App. Math., 30 (1899).

[8] G.S. Bower, Steering and stability of single track vehicles, The Automob. Eng., 5 (1915).

[9] R.H. Pearsall, The stability of the bicycle, Proc. Instr. and Automotive Eng., 17 (1922), pp. 395-402.

[10] M. Kondo, A. Nagaok and F. Yoshimura, Theoretical study on the ruuning stability of twowheelers, Trans. of Soc. Automotive Eng, 17:1 (1963).

[11] R.S. Sharp, The stability and control of motorcycles, J. Mech. Eng. Sc., 13 (1971), pp. 316-329.

[12] R.S. Sharp, The influence of frame flexibility on the lateral stability of motorcycles, J. Mech. Eng. Sc., 16:2 (1974).

[13] R.S. Sharp, Vibrational modes of motorcycles and their design parameter sensitivities, Vehicle NVH and Refinement, Proc. of ICME (1994).

[14] R.S. Sharp and D.J.N. Limebeer, A Motorcycle Model for Stability and Control Analysis, J. of MSD, 6 (2001), pp. 123-142.

[15] V. Cossalter and R. Lot, A motorcycle multibody model for real time simulation based on the natural coordinates approach, J. VSD, 37:6 (2002), pp. 423-447.

[16] S. Hima, L. Nehaoua, N. Seguy and H. Arioui H, Motorcycle Dynamic Model Synthesis for Two Wheeled Driving Simulator, IEEE ITSC, (2007), 812-817.

[17] S. Hima, L. Nehaoua, N. Seguy and H. Arioui, Suitable Two Wheeled Vehicle Dynamics Synthesis for Interactive Motorcycle Simulator, Proc. of IFAC Wor.Cong., Seoul, Korea, (2008).

[18] R. Lot, M. Massaro and V. Cossalter, Advanced motorcycle virtual rider, J. VSD, 46 (2008), pp. 215-224.

[19] R. Lot and V. Cossalter, A nonlinear rider model for motorcycles, Proc. of FISITA Wor. Automotive Cong., Yokohama, Japan, (2006).

[20] R.S. Sharp, S. Evangelou and D.J.N. Limebeer, Advances in the Modelling of Motorcycle Dynamics, J. of MSD, 12 (2004), pp. 251-283.

[21] F. Biral, R. Lot and M. Peretto, Optimization of the Layout of a Racing Motorcycle using the Optimal Maneuver Method, Symp. IAVSD, Berkeley, California, (2007).

[22] A. Saccon, J. Hauser and A. Beghi, A virtual rider for motorcycles: An approach based on optimal control and maneuver regulation, ISCCSP (2008). 
[23] M. Corno, M. Massaro, R. Lot and S.M. Savaresi, On LPV Roll Angle Controller Design for Two-Wheeled Vehicles, Proc. of Bicy. Moto. Dyn., Delft, The Netherlands, (2010).

[24] G. Rill, Simulation von Kraft-fahrzeugen, Vieweg, Braunschweig, Germany, 1994.

[25] S. Zhu, S. Murakami b and H. Nishimura, Motion analysis of a motorcycle taking into account the rider's effects, J. VSD, (2012).

[26] S. Zhu, H. Nishimura and S. Iwamatsu, Dynamical Analysis of Motorcycle by Multibody Dynamics Approach, JSDD, 2 (2008), 703-714.

[27] H.B. Pacejka, Tire and Vehicle Dynamics, Butterworth Heineman Ed., 2005.

[28] S. Evangelou, D.J.N. Limebeer and M.T. Rodriguez, Influence of Road Camber on Motorcycle Stability, ASME J. App. Mech., 75:6, (2008), pp. 231-236.

[29] J.G. Jalon and E. Bayo, Kinematic and Dynamic Simulation of Multibody Systems: The Real-Time challenge, Springer-Verlag, New-York, 1994.

[30] N. Dinga and S. Taherib, An adaptive integrated algorithm for active front steering and direct yaw moment control based on direct Lyapunov method, J. VSD, 48 (2010), pp. 1193-1213.

[31] V. Cossalter, A. Doria and R. Lot, Optimum Suspension Design for Motorcycle Braking, J. VSD, 34 (2000), pp. 175-198.

[32] M. Krstic, I. Kanellakopoulos and P. Kokotovic, Nonlinear and Adaptive Control Design, John Wiley and Sons, 1995.

\section{Appendices}




\section{Notations}

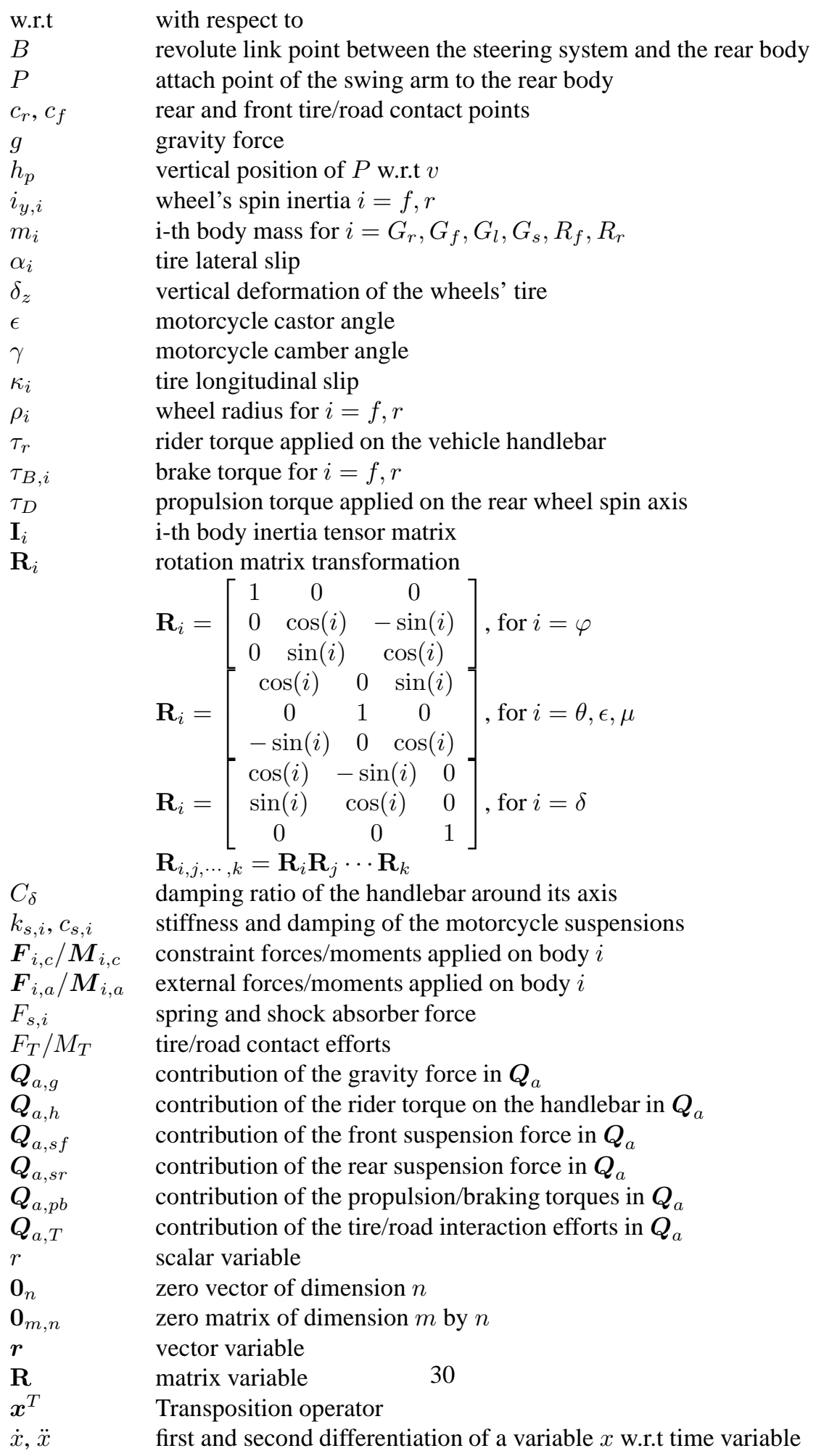




\section{Numerical values}

All numerical values are taken from [20]. However, the coordinate system convention are different (SAE with $z$ axis points downward in [20] and ISO with $z$ axis points upward in this paper). Consequently, the sign of some Pacejka tire parameters must be inverted $\left(p_{K y 1}, p_{K y 6}, p_{K y 7}, q_{B z 10}, q_{D z 3}, q_{D z 8}, q_{D z 9}, q_{E z 2}, q_{E z 5}, q_{H z 3}, q_{H z 4}\right)$.

Table 1: Motorcycle geometric specification (lengths are in [m] and angles in [rad])

\begin{tabular}{cccccc}
\hline$x_{G r}$ & $z_{G r}$ & $x_{G f}$ & $z_{G f}$ & $x_{G l}$ & $z_{G l}$ \\
0.1289 & 0.1116 & 0.0452 & 0.1237 & 0.0679 & -0.263 \\
$x_{G s}$ & $z_{G s}$ & $x_{R r}$ & $z_{R r}$ & $x_{R f}$ & $z_{R f}$ \\
-0.353 & -0.0495 & -0.549 & -0.0638 & 0.0474 & -0.3655 \\
$x_{S l}$ & $z_{S l}$ & $x_{S u}$ & $z_{S u}$ & $h_{p}$ & $l_{P B}$ \\
-0.1047 & -0.1826 & -0.062 & 0.128 & 0.3608 & 0.6831 \\
$\rho_{f}$ & $\rho_{r}$ & $\epsilon$ & & & \\
0.324 & 0.297 & -0.4189 & & & \\
\hline
\end{tabular}

Table 2: Motorcycle mass specification [Kg]

\begin{tabular}{cccccc}
$m_{G r}$ & $m_{G f}$ & $m_{G l}$ & $m_{G s}$ & $m_{R r}$ & $m_{R f}$ \\
165.13 & 9.99 & 7.25 & 8 & 14.7 & 11.9 \\
\hline
\end{tabular}

Table 3: Motorcycle inertia specification $\left[\mathrm{Kg} \cdot \mathrm{m}^{2}\right]$

\begin{tabular}{|c|c|c|c|c|c|c|c|c|}
\hline \multicolumn{3}{|c|}{$\mathbf{I}_{G r}$} & \multicolumn{3}{|c|}{$\mathbf{I}_{G f}$} & \multicolumn{3}{|c|}{$\mathbf{I}_{G l}$} \\
\hline 11.085 & 0 & 3.691 & 1.341 & 0 & 0 & \\
\hline 0 & 22.013 & 0 & 0 & 1.584 & 0 & \multicolumn{2}{|r|}{$\mathbf{0}_{3}$} & \\
\hline 3.691 & 0 & 14.982 & 0 & 0 & 0.4125 & & & \\
\hline & $\mathbf{I}_{G s}$ & & & $\mathbf{I}_{R r}$ & & \multicolumn{3}{|c|}{$\mathbf{I}_{R f}$} \\
\hline 0.02 & 0 & 0 & 0.38 & $3 \quad 0$ & $0)$ & 0.27 & 0 & 0 \\
\hline 0 & 0.259 & 0 & 0 & 0.638 & 0 & 0 & 0.484 & 0 \\
\hline 0 & 0 & 0.259 & 0 & 0 & $0)$ & 0 & 0 & 0 \\
\hline
\end{tabular}

Table 4: Motorcycle handlebar and suspensions specification

\begin{tabular}{ccccccc}
\hline$C_{\delta}\left[\mathrm{N} . \mathrm{m}_{\mathrm{rad}}{ }^{-1} . \mathrm{s}\right]$ & $F_{s, f, 0}[\mathrm{~N}]$ & $F_{s, r, 0}$ & $k_{s, f}\left[\mathrm{~N} \cdot \mathrm{m}^{-1}\right]$ & $k_{s, r}$ & $c_{s, f}\left[\mathrm{~N} \cdot \mathrm{m}^{-1} \cdot \mathrm{s}\right]$ & $c_{s, r}$ \\
6.77 & -796 & 6089.16 & 25000 & 58570 & 2134 & 11650 \\
\hline
\end{tabular}




\section{Linear and angular velocity Jacobian matrices}

Let be the set of coordinates $\boldsymbol{e}_{x}=[1,0,0]^{T}, \boldsymbol{e}_{y}=[0,1,0]^{T}$ and $\boldsymbol{e}_{z}=[0,0,1]^{T}$. The expressions of the linear and angular Jacobian matrices for each body is given by:

- For the rear body:

$$
\begin{aligned}
& \frac{\partial \boldsymbol{v}_{o G_{r}}}{\partial \boldsymbol{\vartheta}}=\left[\begin{array}{lllllll}
\boldsymbol{e}_{x} & \boldsymbol{e}_{y} & \boldsymbol{e}_{z} \times \boldsymbol{r}_{v G_{r}} & \boldsymbol{e}_{z}^{\prime} & \boldsymbol{e}_{x} \times \boldsymbol{r}_{v G_{r}} & \boldsymbol{e}_{\theta} \times \boldsymbol{r}_{P G_{r}} & \mathbf{0}_{3,5}
\end{array}\right] \\
& \frac{\partial \boldsymbol{\omega}_{o G_{r}}}{\partial \boldsymbol{\vartheta}}=\left[\begin{array}{llllll}
\mathbf{0}_{3,2} & \boldsymbol{e}_{z} & \mathbf{0}_{3} & \boldsymbol{e}_{x} & \boldsymbol{e}_{\theta} & \mathbf{0}_{3,5}
\end{array}\right] \\
& \boldsymbol{e}_{z}^{\prime}=\mathbf{R}_{\varphi} \boldsymbol{e}_{z} \quad \boldsymbol{e}_{\theta}=\mathbf{R}_{\varphi} \boldsymbol{e}_{y}
\end{aligned}
$$

- For the front upper body:

$$
\begin{aligned}
& \frac{\partial \boldsymbol{v}_{o G_{f}}}{\partial \boldsymbol{\vartheta}}=\left[\begin{array}{llllllll}
\boldsymbol{e}_{x} & \boldsymbol{e}_{y} & \boldsymbol{e}_{z} \times \boldsymbol{r}_{v G_{f}} & \boldsymbol{e}_{z}^{\prime} & \boldsymbol{e}_{x} \times \boldsymbol{r}_{v G_{f}} & \boldsymbol{e}_{\theta} \times \boldsymbol{r}_{P G_{f}} & \boldsymbol{e}_{\delta} \times \boldsymbol{r}_{B G_{f}} & \mathbf{0}_{3,4}
\end{array}\right] \\
& \frac{\partial \boldsymbol{\omega}_{o G_{f}}}{\partial \boldsymbol{\vartheta}}=\left[\begin{array}{lllllll}
\mathbf{0}_{3,2} & \boldsymbol{e}_{z} & \mathbf{0}_{3} & \boldsymbol{e}_{x} & \boldsymbol{e}_{\theta} & \boldsymbol{e}_{\delta} & \mathbf{0}_{3,4}
\end{array}\right] \\
& \boldsymbol{e}_{\delta}=\mathbf{R}_{\varphi, \theta, \epsilon} \boldsymbol{e}_{z}
\end{aligned}
$$

- For the front lower body:

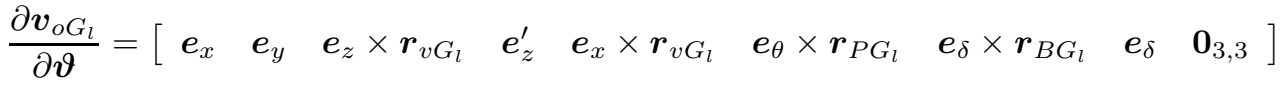

$$
\begin{aligned}
& \frac{\partial \boldsymbol{\omega}_{o G_{l}}}{\partial \boldsymbol{\vartheta}}=\left[\begin{array}{lllllll}
\mathbf{0}_{3,2} & \boldsymbol{e}_{z} & \mathbf{0}_{3} & \boldsymbol{e}_{x} & \mathbf{0}_{3} & \boldsymbol{e}_{\delta} & \mathbf{0}_{3,4}
\end{array}\right]
\end{aligned}
$$

- For the swing arm body:

$$
\begin{aligned}
& \frac{\partial \boldsymbol{v}_{o G_{s}}}{\partial \boldsymbol{\vartheta}}=\left[\begin{array}{llllllll}
\boldsymbol{e}_{x} & \boldsymbol{e}_{y} & \boldsymbol{e}_{z} \times \boldsymbol{r}_{v G_{s}} & \boldsymbol{e}_{z}^{\prime} & \boldsymbol{e}_{x} \times \boldsymbol{r}_{v G_{s}} & \mathbf{0}_{3,3} & \boldsymbol{e}_{\mu} \times \boldsymbol{r}_{P G_{s}} & \mathbf{0}_{3,2}
\end{array}\right] \\
& \frac{\partial \boldsymbol{\omega}_{o G_{s}}}{\partial \boldsymbol{\vartheta}}=\left[\begin{array}{lllllll}
\mathbf{0}_{3,2} & \boldsymbol{e}_{z} & \mathbf{0}_{3} & \boldsymbol{e}_{x} & \mathbf{0}_{3,3} & \boldsymbol{e}_{\mu} & \mathbf{0}_{3,2}
\end{array}\right] \\
& \boldsymbol{e}_{\mu}=\boldsymbol{e}_{\theta}
\end{aligned}
$$

- For the rear wheel:

$$
\begin{aligned}
& \frac{\partial \boldsymbol{v}_{o R_{r}}}{\partial \boldsymbol{\vartheta}}=\left[\begin{array}{llllllll}
\boldsymbol{e}_{x} & \boldsymbol{e}_{y} & \boldsymbol{e}_{z} \times \boldsymbol{r}_{v R_{r}} & \boldsymbol{e}_{z}^{\prime} & \boldsymbol{e}_{x} \times \boldsymbol{r}_{v R_{r}} & \mathbf{0}_{3,3} & \boldsymbol{e}_{\mu} \times \boldsymbol{r}_{P R_{r}} & \mathbf{0}_{3,2}
\end{array}\right] \\
& \frac{\partial \boldsymbol{\omega}_{o R_{r}}}{\partial \boldsymbol{\vartheta}}=\left[\begin{array}{llllll}
\mathbf{0}_{3,2} & \boldsymbol{e}_{z} & \mathbf{0}_{3} & \boldsymbol{e}_{x} & \mathbf{0}_{3,5} & \boldsymbol{e}_{\zeta_{r}}
\end{array}\right] \\
& \boldsymbol{e}_{\zeta_{r}}=\boldsymbol{e}_{\theta}
\end{aligned}
$$


- For the front wheel:

$$
\begin{aligned}
& \frac{\partial \boldsymbol{v}_{o R_{f}}}{\partial \boldsymbol{\vartheta}}=\left[\begin{array}{lllllllll}
\boldsymbol{e}_{x} & \boldsymbol{e}_{y} & \boldsymbol{e}_{z} \times \boldsymbol{r}_{v R_{f}} & \boldsymbol{e}_{z}^{\prime} & \boldsymbol{e}_{x} \times \boldsymbol{r}_{v R_{f}} & \boldsymbol{e}_{\theta} \times \boldsymbol{r}_{P R_{f}} & \boldsymbol{e}_{\delta} \times \boldsymbol{r}_{B R_{f}} & \boldsymbol{e}_{\delta} & \mathbf{0}_{3,3}
\end{array}\right] \\
& \frac{\partial \boldsymbol{\omega}_{o R_{f}}}{\partial \boldsymbol{\vartheta}}=\left[\begin{array}{lllllllll}
\mathbf{0}_{3,2} & \boldsymbol{e}_{z} & \mathbf{0}_{3,1} & \boldsymbol{e}_{x} & \mathbf{0}_{3,1} & \boldsymbol{e}_{\delta} & \mathbf{0}_{3,2} & \boldsymbol{e}_{\zeta_{f}} & \mathbf{0}_{3}
\end{array}\right] \\
& \boldsymbol{e}_{\zeta, f}=\mathbf{R}_{\varphi, \theta, \epsilon, \delta} \boldsymbol{e}_{y}
\end{aligned}
$$

